\title{
Evidence suggests children are being left behind in COVID-19 mental health research
}

\author{
Nicole Racine ${ }^{1,2}$. Daphne J. Korczak ${ }^{3,4}$ - Sheri Madigan ${ }^{1,2}$
}

Received: 8 October 2020 / Accepted: 23 October 2020 / Published online: 5 November 2020

(c) Springer-Verlag GmbH Germany, part of Springer Nature 2020

Keywords Mental health $\cdot$ Children · COVID-19

The prevalence of mental health difficulties during COVID19 has increased [6], especially among vulnerable groups, including children $[2,4]$. Indeed, the most considerable social and economic burden of COVID-19 will likely fall to the youngest members of society, leaving a generation vulnerable to higher rates of mental health difficulties [1]. While the manifestation and medical symptoms of COVID19 have been mild in children, the disruption to their daily lives has been unprecedented, from extended school closures, shut-down of extra-curricular activities and supports, and isolation from friends and exposure to increased family stress and violence. The presence of any one of these risk factors in a child's life has negative implications for mental health, including anxiety and depressive symptoms, and due to COVID-19, some children are experiencing them simultaneously, with multiplicative impact. Moreover, COVID-19 and its related disruptions are projected to persist well into 2021, with many children remaining out of school and largescale shutdowns continuing. All told, COVID-19 is a ripe context for a dramatic surge in mental health difficulties in children.

To avoid potentially catastrophic outcomes for children around the world, the United Nations has identified the need for "a rapid accumulation of data on the scale and nature of impacts among children" [5]. Yet, when we examine the

Sheri Madigan

sheri.madigan@ucalgary.ca

1 Department of Psychology, University of Calgary, Calgary, $\mathrm{AB}$, Canada

2 Alberta Children's Hospital Research Institute, Calgary, AB, Canada

3 Department of Psychiatry, Hospital for Sick Children, Toronto, ON, Canada

4 Department of Psychiatry, University of Toronto, Toronto, ON, Canada amount of mental health research involving children since the onset of COVID-19, there is a striking lack of research. To inform policy and practice, our research group conducted a large-scale search across 5 research databases (PsycINFO, Cochrane Central Register of Controlled Trials, EMBASE, and MEDLINE) to identify the prevalence of mental health difficulties in children and adults since COVID-19. After reviewing 3,405 abstracts, we found 73 independent studies documenting the prevalence of depression, anxiety, and stress in adults, compared to 6 studies in child populations [6]. This means that at the time of the search, the ratio of adults to child mental health studies since COVID-19 is $12: 1$. While $25 \%$ of the world's population are children, they make up only $\sim 8 \%$ of research being conducted on the mental health impacts of COVID-19. Child mental health research is drastically lagging behind research on adults, and there is an urgent need to prioritize funding for research initiatives that explicitly examines children's mental health.

There are several reasons why the mental health of children needs to be prioritized during COVID-19. First, the social and contextual implications of the pandemic have been devastating for many children. School closures have not only stripped many children of one of their primary social supports and mental health resources but have also exacerbated social and economic inequalities, particularly for children who are at most significant risk for developing mental health difficulties. Second, there is strong evidence that childhood, particularly early childhood, is a sensitive period for exposure to stress. Exposure to high levels of stress in childhood can alter the way individuals respond to stress later in life and predispose them to health and mental health difficulties. Research that identifies which factors and how these factors protect against the negative mental health impacts of COVID-19 will be critical. Third, children are not little adults and the factors that contribute to their mental health will differ from those that impact adults. 
There is a need to understand the specific processes and mechanisms that impact children's mental health and the best way to intervene. Finally, it is well established via the Heckman Equation that upstream investments in early childhood, especially in the most disadvantaged groups, provide the highest rate of return on investments. Prioritizing child mental health research is in the best interest of our long-term economic prosperity.

Why is there a disparity in child health and mental health research? Despite the evidence that the groundwork for mental health and wellbeing across the lifespan is established in childhood, child health research has been notoriously underfunded [3]. There is also generally poor access to child mental health treatment, with 50-66\% of children in developed countries failing to receive the mental health treatment they need $[7,8]$. Both disparities in child mental health research and service access are problematic, particularly in the face of COVID-19, which will increase the need for innovative solutions to decrease the mental health burden for children. Although we anticipate that child mental health researchers worldwide have heeded the call to conduct research that will inform child mental health policy and practice, the chronic underfunding of child mental health research makes this process onerous and can result in delays in research execution as a result.

To date, the available evidence on the impact of COVID19 and its associated mitigation strategies on child and adolescent mental health is scarce [6]. The research that does exist has several limitations that make it difficult to draw meaningful conclusions, including a lack of comparative baseline data, a lack of child-report, non-representative samples, cross-sectional surveys, and unclear reporting of diagnostic symptomatology. As a result of these limitations, the call to policy to support children's mental health falls flat. Methodologically rigorous studies that provide estimates that can be compared to baseline data, conducted in samples that are representative of national populations, using validated tools, compare populations exposed to varying levels of mitigation strategies, and if possible, conduct diagnostic interviews are needed. Notably, some children have shown remarkable resilience in the midst of COVID19, and this also warrants concerted study. How have some children prevailed despite the removal of critical sources of support? What positive supports have they relied upon to overcome this hardship?

Children are some of the most vulnerable members of our society yet invisible casualties of this pandemic. They count on adults to be their advocates. Children depend on us to notice and act on the inequalities they are facing. Our collective responsibility is to give them a voice by ensuring that their mental health is an international priority, both within and outside the academic realm.

\section{Availability of data}

Not applicable.

Author contributions NR, SM, DK: writing — original draft, writingreview and editing.

Funding Research support was provided to Dr. Madigan by the Canada Research Chairs program. Dr. Racine was supported by a Postdoctoral Trainee Award from Alberta Innovates. The funding sources had no role in the current manuscript.

\section{Compliance with ethical standards}

Conflicts of interest None to declare.

Ethics approval N/A.

\section{References}

1. Clemens V, Deschamps P, Fegert JM, Anagnostopoulos D, Bailey S, Doyle M, Eliez S, Hansen AS, Hebebrand J, Hillegers M, Jacobs B, Karwautz A, Kiss E, Kotsis K, Kumperscak HG, Pejovic-Milovancevic M, Christensen AMR, Raynaud JP, Westerinen H, Visnapuu-Bernadt P (2020) Potential effects of "social" distancing measures and school lockdown on child and adolescent mental health. Eur Child Adolesc Psychiatry 29:739-742

2. Dvorsky MR, Breaux R, Becker SP (2020) Finding ordinary magic in extraordinary times: child and adolescent resilience during the COVID-19 pandemic. Eur Child Adolesc Psychiatry 1:1-3

3. Flores G, Lesley B (2014) Children and U.S. federal policy on health and health care: seen but not heard. JAMA Pediatr 168:1155-1163

4. Jefsen O, Rohde C, Norremark B, Ostergaard S (2020) Editorial perspective: COVID-19 pandemic-related psychopathology in children and adolescents with mental illness. J Child Psychol Psychiatry. https://doi.org/10.1111/jcpp.13292

5. United Nations (2020) Policy brief: the impact of COVID-19 on children

6. Racine N, Cooke JE, Eirich R, Korczak DJ, McArthur B, Madigan S (2020) Child and adolesent mental illness during COVID-19: A rapid review. Psychiatry Res. https://doi.org/10.1016/j.psych res.2020.113307

7. Waddell C, Georgiades K, Duncan L, Comeau J, Reid GJ, O'Briain W, Lampard R, Boyle MH, Ontario Child Health Study T (2019) 2014 Ontario child health study findings: policy implications for Canada. Can J Psychiatry 64:227-231

8. Whitney DG, Peterson MD (2019) US national and state-level prevalence of mental health disorders and disparities of mental health care use in children. JAMA Pediatr 173:389-391 